\title{
Eco formación del docente universitario y su modelo ecológico de la universidad de Guayaquil
}

\section{Eco Training of the university professor and his ecological model of the university of Guayaquil}

Mgs. Norma Narcisa Garcés Garcés

Universidad de Guayaquil, Ecuador

MSc Camila Orellana Lara

Ing. Mario Pesantes Torres

Ing. Génesis Salazar Garcés

Universidad de Especialidades Espíritu Santo, Ecuador

Autor para correspondencia: norma.garcesg@ug.edu.ec, norma.garcesg@gmail.com, vimapeto@hotmail.com, vimapeto@ hotmail.com, gsalazarg@uees.edu.ec

Fecha de recepción: 20 de agosto de 2018 - Fecha de aceptación: 01 de Diciembre de 2018

Resumen: La eco-formación de la educación en el docente universitario concibe una reforma del pensamiento, una necesidad democrática, desde una comprensión ecológica integrada del ser humano que responde a los problemas del tiempo; por lo que es esencial el manejo de herramientas que permitan la autonomía, la reflexión que requiere examinar y hacer una autocrítica de la mente. El punto de vista considera como parte del ecosistema, en su ambiente y en todos los sistemas que integra, la familia, la sociedad, la comunidad, los docentes, y todas las personas que se encuentran durante toda la vida. Educar en conciencia, valores y emociones, por la acción sensata y responsable, tiene cambios interiores y exteriores que muestran como interactuar en una convivencia armónica con el mundo, conociendo la naturaleza humana, que contribuye a una mejor calidad y estilo de vida. La educación relaciona actividades académicas típicas con el medio ambiente, multiculturalidad, desarrollo humano y urbano, bajo el apoyo de las entidades educativas, aplicadas en países subdesarrollados. La investigación que se aplico fue la documental y entrevistas a expertos para conocer el involucramiento de estos temas en malla académica y como se enlazan las materias a estos tópicos.

Palabras clave: eco-formación; medio ambiente; multiculturalidad; relaciones transdisciplinarias

Abstract: The eco-formation of education in the university teacher conceives a reform of thought, a democratic need, from an integrated ecological understanding of the human being that responds to the problems of time; therefore, it is essential to use tools that allow autonomy, reflection that requires examining and making a self-criticism of the mind. The point of view considers as part of the ecosystem, in its environment and in all the systems that it integrates, the family, the society, the community, the teachers, and all the people who are throughout their lives. Therefore, it consists in educating in conscience, values and emotions, by sensible and responsible action, it has internal and external changes that show how to interact in a harmonious coexistence with the world, knowing human nature, which contributes to a better quality and Lifestyle. Education links typical academic activities with the environment, multiculturalism, human and urban 
development, with the support of educational entities, applied in underdeveloped countries. The research that was applied was the documentary and interviews to experts to know the involvement of these topics in academic mesh and how the subjects are linked to these topics.

Key words: greening; environment; multiculturalism; transdisciplinary relationships

\section{Introducción}

En la actualidad, el sistema educativo se plantea de forma individualista por lo cual está basado en que el individuo sea parte del medio laboral únicamente basándose en sus propias capacidades y habilidades, motivo por el cual, la nueva propuesta que se está implementando comienza por el trabajo colectivo y una visión ecologizada de la educación, la cual forma parte de una estrategia a seguir acorde a los nuevos tiempos enfocándose en el desarrollo integral docente y luego en el del estudiante, el cual considera al primero como un líder de opinión.

La propuesta de cambios en la reforma educativa no es algo nuevo, por lo que ha sido eje de discusión en múltiples ocasiones, en las cuales muchos estudiosos proponen métodos que otorguen más independencia a los individuos mediante una educación personalizada y otros proponen modificaciones dentro de la educación tradicional mediante la unificación de la misma.

Según (Miller, 2010), indica:

Reconozcamos que el enfoque holístico es esencialmente un enfoque ecológico. El respeto por lo más profundo del ser humano involucra necesariamente el respeto por la Naturaleza y por la Tierra: es el respeto por la vida toda. La educación holística desafía la visión materialista, mecanicista e intelectualista de la era industrial, ya que mientras consideremos que la tierra es un recurso para ser explotado y controlado, lo mismo sentiremos con respecto a nuestros niños (pág. 3)

La ecologización de la educación está concebido desde una comprensión ecológica que el ser humano es multidimensional e interdependiente. Por ello, lo concibe como parte del ecosistema, en su ambiente, en todos los sistemas que lo integra y la sociedad. Esto permite mantener una cultura de respeto, valores y conciencia en emociones de parte de todos los involucrados. Educar de forma consciente y responsable es enseñar al ser humano, debido a que produce cambios interiores y exteriores nos muestra la forma de relacionarnos armónicamente con el mundo, conociendo y desarrollando nuestra naturaleza humana, afectando nuestra calidad y estilo de vida, contribuyendo al cuidado del planeta que es nuestro hogar.

Al ecologizar la educación se propone articular campos temáticos que se relacionan unos con otros con el propósito de que se desarrollen de forma conjunta, lo que representa una oportunidad para enlazar los procesos educativos con el medio ambiente, desarrollo rural, derechos humanos y multiculturalidad. Organismos como la UNESCO y la ONU han sido participes de este desarrollo sostenible en países en vías de desarrollo en los cuales aún existe un gran nivel de analfabetismo en la población. Este nuevo sistema educativo requiere a su vez las aportaciones tanto de padres, como de docentes, directivos y alumnos.

La primera aportación de la UNESCO (2005) respecto al tema buscaba modificar el aprendizaje de las ciencias para que el estudiante disponga de un mínimo de conocimientos 
necesarios exigidos por la sociedad, en los que se demanda un personal humano mayor capacitado que hace medio siglo, formando de esta manera a un ciudadano responsable, competitivo, colaborador y critico ante los avances tecnológicos y científicos.

Según (López, 2013), indica en uno de sus blog:

La educación científica es la capacidad de entender temas elementales de ciencia a un nivel suficiente para participar en el debate científico. Aunque intuitivamente todos aceptamos los beneficios de estar educado científicamente, cabe preguntar cuántos de nosotros realmente estamos lo suficientemente informados en cuestiones de ciencia.

La educación científica por su lado se basa en la especialización en una rama en Occidente, considerándose este como parte del saber y sin mucho debate detrás del mismo más que la comprobación de una teoría existente. Múltiples contenidos académicos han sido desechados en estas parcelas del saber, lo que consigue tanto desinterés de parte del docente como del estudiante por entender o interesarse en conseguir más información de estos conocimientos. Esto llega a ser aún más evidente en áreas como Física, Química, Matemáticas y ciencias derivadas de las mismas.

Actualmente el Ecuador cuenta con un bachillerato unificado que prepara a los estudiantes para la vida laboral, su inclusión social y el emprendimiento, además de un examen general realizado por la SENECYT para ubicar a los alumnos dentro de una carrera y universidad de acuerdo al puntaje que obtengan. Las universidades a su vez han tenido que modificar sus programas de estudio a los constantes cambios en las exigencias laborales.

Mientras que la SENECYT, para mejorar la calidad de la educación, en búsqueda de la eficiencia ha realizado evaluaciones en las distintas universidades del país para colocarlas en una categoría de la A a la E, siendo la primera las mejores universidades del país y con mejor infraestructura, y las ultimas las que son nuevamente llevadas a evaluación después de realizados cambios para decidir si continúan en funcionamiento.

Por lo cual la ecologización de la educación es fundamental en el sistema universitario al concebir una comprensión integrada sobre el ser humano como multidimensional e independiente, con el propósito de integrarlo en la sociedad y que pueda contribuir con conocimientos en la misma. Esto permite un mayor involucramiento de las personas con las que interactúa en diversos espacios e incluso el laboral.

Según, (Spaargaren,Mol y Buttel , 2000) indica: "Si los diferentes grupos ecologistas y sus propuestas eco utópicas han fracasado en su intento de ecologización del mundo, veamos a continuación qué papel ha desarrollado la ética ecológica en esta decepción”.

Para educar en la ecologización se consideran de gran importancia los valores y las emociones, con los cuales el ser humano se vuelve más consciente y responsable de sus contribuciones. Estos cambios se han realizado tanto de manera interna como externa por parte de las instituciones académicas, las cuales ahora no solo se enfocan en la enseñanza básica sino además en desarrollar la naturaleza humana afectando el estilo y calidad de vida de las personas a modo de contribución para el planeta. 
Según Miller (1990), el enfoque holístico es reconocido como ecológico, que involucra además a la naturaleza y a la vida, desafiando la visión materialista de la era industrial y al mismo capitalismo al poner un alto a la sobreexplotación descarada de recursos importantes para la sobrevivencia en el mundo o a la destrucción de los mismos. Pero a estos intereses altruistas, se enfrenta el problema de la búsqueda de soluciones a problemas contemporáneos y a la mutación de los mismos por la demanda académica presente y futura. Estas demandas pueden ser culturales, políticas, sociales y económicas dependiendo de la región en la que se encuentre la institución, además del problema de adaptabilidad de parte de hombres y mujeres a un sistema desconocido para ellos.

Según, (ODOM Y KAISER, 1997) indica: "En nuestro caso diremos que el modelo ecológico de Bronfenbrener supone que, como mínimo, hemos de tener en cuenta los apoyos sociales de que disponen los padres, sus necesidades, e incluso sus estilos familiares".

En cuanto a la elaboración y aplicación de la estrategia de ecologización lleva como base los siguientes principios:

- La interdependencia como parte de la conciencia ecológica que reside en la reintegración del medio ambiente en la conciencia antroposocial, en las ideas de biosfera y ecosistema donde se aprecian los aspectos positivos.

- La perspectiva eco-sistémica que está enfocada a la comprensión de que nuestro medioambiente es producto de factores ecológicos, culturales, económicos y sociales.

- En la visión psico/socio/emocional/ambiental se incorpora la reflexión y discusión sobre los impactos de las emociones, pensamientos y acciones humanas en nuestra propia vida y el medio ambiente, eliminando la brecha entre nosotros y la naturaleza.

- La neurociencia aplicada a la educación mediante herramientas valiosas para mejorar la calidad de la educación, el desarrollo humano y delinear estrategias efectivas para el aprendizaje y la enseñanza.

- La educación en emociones mediante charlas y conferencias a docentes para promover la conciencia eco-sistémica con intención de cooperación medioambiental del individuo con su entorno (estudiantes).

- El trabajo en equipo, comunicación y desarrollo del potencial creativo, enfocándose en las fortalezas personales y grupales.

Según, (Morin, 1996) nos indica:

Se conoce por Interdisciplinar la forma de organización de los conocimientos, donde los métodos que han sido utilizados con éxito dentro de una disciplina, se transfieren a otra, introduciéndolos en ella sobre la base de una justificación, que pretende siempre una ampliación de los descubrimientos posibles o la fundamentación de estos.

En estas relaciones transdisciplinarias podemos ejemplificar casos como el de la relación entre la educación y la salud la cual enseña a escucharse a sí mismo, a educar en emociones, e iniciar una conciencia ecológica basada en el cuidado y la cooperación medioambiental, en pos de la armonía de la persona con el entorno familiar, ambiental y social. $\mathrm{O}$ el de la neurociencia la cual estudia la influencia de los estímulos que se le dé al individuo y como alcanzan un 
desarrollo equilibrado a través de sus 3 cerebros (racional, emocional reptil) y alcanza un pensamiento crítico frente a posibles soluciones al problema debido a que una cultura ecológica requiere de participación consciente y responsable de los integrantes de instituciones académicas.

Mientras entre los pilares que sostienen esta ecologización tenemos la inteligencia emocional como factor fundamental del bienestar y calidad humano, la ecología emocional referente a un paralelismo entre los conflictos de las personas en el mundo actual y los problemas ecológicos, la incidencia metodológica del medio ambiente, la importancia de una educación a favor del medioambiente mediante un componente ético que incide en la formación de valores. Así como, el desarrollo de competencias y talentos enfatizando en el desarrollo potencial de ambos hemisferios cerebrales, y aplicados a la inteligencia emocional y la experiencia participativa y asociativa de docente- estudiantes.

El docente también se enfrenta a nuevas competencias, entre las cuales comprende:

- La democracia como forma de ejercer el derecho a la elección de una autoridad con el fin de mejorar la sociedad.

- Las libertades incondicionales del ser humano respecto a sus decisiones

- Ambientes de aprendizaje significativos como aquellos correspondientes al cambio climático y las ciencias tradicionales desde un enfoque critico

- Pedagogías innovadoras a la educación tradicional

- Otorgar una educación de calidad, inclusiva y equitativa de acuerdo a las exigencias socio-culturales

El primer cambio que puede realizar el docente es el de mejorar la relación entre docenteestudiante con un ambiente académico agradable en el cual se aprende a comprender nuestro propio mundo, para vincularnos y actuar positivamente con los demás. Minimizando de esta manera, problemas como la fatiga, el estrés, enfermedades psíquicas, explosiones emocionales, agresividad e infelicidad; transformando estos estados negativos en oportunidades para desarrollarnos como personas y nuestras relaciones interpersonales.

\section{Modelo educativo ecológico de la Universidad de Guayaquil}

En América Latina, la globalización ha provocado cambios económicos, culturales, sociales, políticos, y de intereses en los gobiernos; estos procesos han hecho que la vida del ser humano transcurra mucho más rápido porque las nuevas tecnologías han hecho que las actividades se las realicen tan rápido y el ser humano tenga que trabajar de una forma acelerada, lo que ha perjudicado en tiempos de descanso y tranquilidad trayendo como consecuencia el estrés y múltiples enfermedades para los docentes y la población en general.

Por lo tanto, las tendencias en educación superior plantean un sistema más equitativo, una mayor diferenciación y diversificación, alineados a las necesidades del desarrollo económico y social del Ecuador. En consecuencia, la Universidad de Guayaquil promueve nuevos perfiles de egreso como talento humano formado en ciencia, investigación y valores, haciendo posible la gestión social del conocimiento. 
El desafío de la educación superior a nivel global radica en aportar construcciones del conocimiento para transformar los modelos económicos, matriz productiva del país, fortalecer el mercado, los sistemas de competitividad, la educación en valores, problemas de la pobreza, la exclusión del trabajo a los mismos jóvenes y a los adultos mayores, la crisis ecológica, la salud y el acceso a la educación. La innovación por su lado, juega un papel muy importante en la incorporación del valor social del conocimiento, la comprensión de nuevas infraestructuras de integración del saber y la constitución de colectivos de inteligencia estratégica que serían los encargados de crear planes y proyectos novedosos que aporten al desarrollo del país.

\section{La transformación del sistema educativo}

La educación es y será un medio para el desarrollo personal del ser humano y crecimiento económico del país, la clave es mejorar la equidad social, crear fuentes de trabajo para los profesionales, no profesionales, técnicos, y hasta para los no alfabetizados, porque cada uno de los individuos cuenta con facultades o dones que puede desempeñarse en el ámbito que sea entrenado o instruido, por lo tanto, en esta sociedad no debería existir la pobreza, ni pobres mendigando en la calle. Los grandes empresarios deben analizar y repensar que la fuerza laboral es necesaria para el desarrollo de su empresa y de su país.

El desarrollo científico y tecnológico y la generación y difusión de las investigaciones son necesarias en el siglo XXI porque producen nuevos conocimientos, técnicas, procesos de enseñanza- aprendizaje que promueven la formación de profesionales

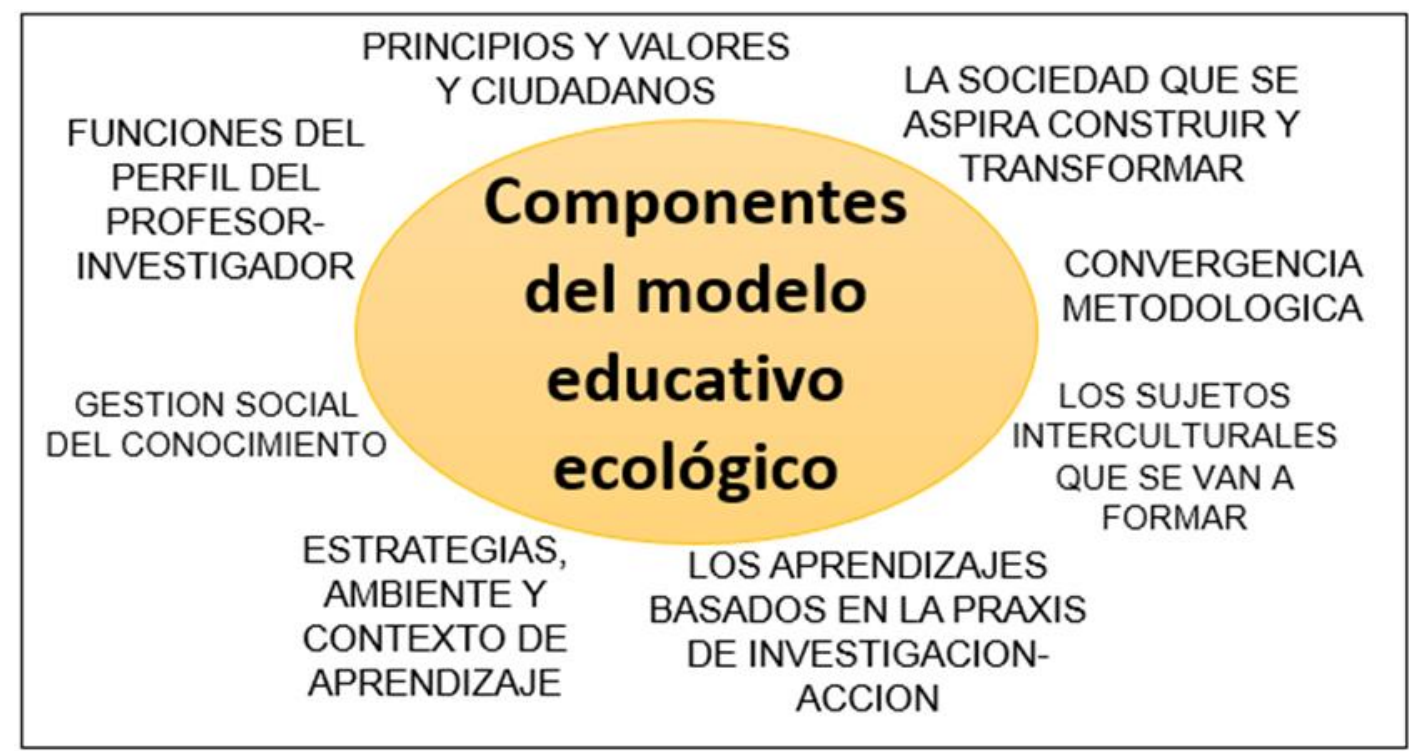

Fig. 1. Componentes del modelo educativo ecológico

Fuente: Larrea (2016)

\section{Objetivo general}

Contribuir al desarrollo de un sistema educativo, científico, de educación en valores y emocional para lograr una comprensión ecológica del mundo más definida, afectiva e integradora en el ecosistema, seres humanos, para mejorar la calidad de vida. 


\section{Objetivos específicos}

$\checkmark$ Fomentar la comunicación ecológica, afectiva, humana con el ecosistema.

$\checkmark$ Construir un mundo de paz, solidario y sostenible a través de la convivencia social

$\checkmark$ Análisis y discusiones

Con el propósito de ecologizar la formación del docente universitario, los centros educativos están en la labor de garantizar oportunidades de aprendizaje para el desarrollo de una comunidad en pos de la innovación y la solidaridad, viéndose estos más comprometidos en cubrir las necesidades humanas y sociales sin destruir el planeta, la aplicación de la misma corresponde a transformaciones económicas, políticas, ecológicas, productivas y sociales.

Para lo cual, incorpora la creación de ambientes estimulantes que faciliten el interés en el aprendizaje, la reutilización y modificación de elementos ecológicos, el uso de elementos mediáticos (fotos, videos, sonidos), elementos vivenciales de la naturaleza que despierten la conciencia ecológica, información sobre estilos de vida sostenibles; y la creación de mallas de educación superior que den asignaturas en valores, ética profesional, equidad, inclusión y el respeto a la justicia social. Las mismas siendo ignoradas en mejora de la técnica, que otorga empleados más competitivos y productivos, considerada únicamente valiosa la información que genera dinero u obedece a una oferta del mercado laboral.

Por lo cual es clave la integración de nuevos enfoques para la formación de los docentes en servicio, así como una visión emocional y socio-ambiental en la pedagogía y el resto de carreras profesionales. En Ecuador este es un tema al que se le ha restado importancia por lo que las acciones son mínimas contrario a otros países de la región como Brasil, Venezuela y Chile, instalados de manera no formal en la educación formal.

\section{Materiales y métodos}

Para este estudio se realizó una investigación documental, bajo la modalidad descriptiva, por ser un tema de análisis reciente en el país que se ha desarrollado mayormente a través de la formación extracurricular, motivo por el cual las mallas académicas están adaptadas a los nuevos requerimientos del mercado laboral ecuatoriano.

Se hizo un estudio documental basado en libros, guías, informes, artículos científicos de un tema que ha sido poco explorado en el pasado (desde inicios del 2011 hasta el 2014) debido a que recientemente las universidades se están acogiendo a las reglamentaciones para establecer la excelencia académica mediante la SENECYT, además de recoger información sobre las funciones de la universidad mediante entrevistas a los docentes y a los estudiantes sobre el impacto laboral, productivo y social que se ha producido cuando obtenido el título de licenciado buscan ofertas de empleo.

Se realizaron alrededor de 2-3 preguntas rápidas con facilidad para ser contestadas en muy poco tiempo a 12 personas sobre sus expectativas al salir de la universidad, la razón por la que escogieron esta carrera y la percepción social de la obtención de un título de la UG. 
Se realizó un análisis estadístico mediante muestreo aleatorio estratificado dentro de la facultad a un grupo de estudiantes de los últimos años y a 3 de sus profesores. De la cual se obtuvo los resultados cualitativos más relevantes como una opinión más cercana a la general. El tiempo que duro este trabajo fue de 4 días en los que se citó a los alumnos y docentes a un aula donde no tuvieran distractores.

\section{Resultados}

La transformación académica en el Ecuador, y especialmente en la UG a partir del año 2015 ha realizado cambios importantes en los procesos educativos, la gestión administrativa y la forma de concebir la organización del conocimiento y los saberes en orientación a cumplir espacios sociales que desarrollen emprendimientos organizados para el cambio de las realidades y los problemas sociales y comunes de la sociedad.

Las mallas curriculares y la reforma de los modelos académicos, se integraron a las nuevas tendencias mundiales y de control. Las necesidades del país y la articulación con el modelo de desarrollo económico. Las matrices de la organización de la academia son:

- La organización del conocimiento, en la que se integran los dominios científicos, tecnológicos y humanísticos.

- La organización de los aprendizajes, nivel técnico, tecnológico, de grado y posgrado, que posibilite itinerarios de formación del talento humano en base a campos de estudio pertinentes con las necesidades nacionales y locales.

- La organización académica, orientada al fortalecimiento epistemológico e institucional del sistema educativo superior y de las instituciones de educación superior que lo constituyen, así como en la formación de redes académicas y del conocimiento que impulsan capacidades y potencialidades en las funciones de investigación, formación y vinculación con la sociedad.

\section{Conclusiones}

El papel de la formación universitaria (conjuntamente con el modelo ecológico de la Universidad de Guayaquil) consiste en desarrollar oportunidades con redes de investigación que involucran a sus actores a desarrollar un determinado objetivo y orientar esfuerzos, intereses y recursos humanos y financieros enfocados a una línea de investigación conjunta, para lograr resultados con una política de desarrollo científico, social, tecnológico e innovador en cualquier área del conocimiento.

El modelo educativo de la Universidad de Guayaquil promueve la excelencia académica y metodologías del aprendizaje sustentados en la integración de saberes, y la investigación, difusión científica.

\section{Recomendaciones}


Motivar a los docentes para crear redes de investigación y promover reformas educacionales para darle sentido al cambio educativo. La formación y el desarrollo profesional son relevante en el académico, porque las buenas prácticas de la enseñanza- aprendizaje en el aula es un recurso innovador que permite mejorar la educación. Los contextos donde se desenvuelven es una buena forma de investigar y transformar a través de narrativas que sirvan para crear situaciones, procesos, experiencias educativas con un fin.

\section{Bibliografía}

Blanco, N. (2010). Política Curricular Y Prácticas Educativas. Documento Policopiado. Programa De Master- Doctorado En Políticas Y Prácticas De Innovación Educativa Para La Sociedad Del Conocimiento. Universidad De Almería- Ucsg. Guayaquil- Ecuador.

Bronfenbrenner, U. (1987). La Ecología Del Desarrollo Humano. Barcelona: Paidós.

Carrión, J. (2010). La Guía Docente: ¿Burocracia O Reflexión? Documento Policopiado. Master Doctoral En Políticas Y Prácticas De Innovación Educativa Para La Sociedad Del Conocimiento Universidad De Almería- Ucsg. Guayaquil- Ecuador.

Conea. (2009). Evaluación Del Desempeño Institucional De Las Universidades Y Escuelas Politécnicas Del Ecuador. Quito: Conea.

Conferencia Regional De Educación Superior Cres. (2008). Declaración Y Plan De Acción De La Conferencia Regional De Educación Superior En América Latina Y El Caribe. Instituto Internacional De La Unesco Para La Educación Superior En América Latina Y El Caribe Iesalc. Barcelona: Ministerio De Ciencia E Innovación De España.

Gottifredi. (1996). Una Forma De Profundizar La Relación Universidad- Sociedad. Obtenido De Http://Www.Scielo.Org.Ar/Pdf/Cfhycs/N15/N15a03.Pdf

Larrea, E., \& Montalván, M. (2016). Modelo Educativo Ecológico De La Universidad De Guayaquil. Modelo De Formación Integral Orientado A La Investigación Y Gestión Social Del Conocimiento. Guayaquil, Guayas, Ecuador.

López, J. C. (21 De Febrero De 2013). Cuaderno De Cultura Científica. Obtenido De La Importancia De La Educación Científica, Por Juan Carlos López: Https:/Culturacientifica.Com/2013/02/21/La-Importancia-De-La-Educacion-CientificaPor-Juan-Carlos-Lopez/

Miller, R. (13, 14,15 De Septiembre De 2010). Metas Educativas 2021. Obtenido De Ecologización De La Educación: "Una Estrategia Educativa Hacia El 2021": Http: //Www.Adeepra.Org.Ar/Congresos/Congreso\%20iberoamericano/Metas2021/Rle2314_L ares.Pdfmorin, E. (S.F De S.F De 1996). El Padre Del Pensamiento Complejo. Obtenido De ¿Qué Es Transdisciplinariedad?: Http://Edgarmorinmultiversidad.Org/Index.Php/QueEs-Transdisciplinariedad.Html 
Morín, E. (2002). El Método. El Conocimiento Del Conocimiento. Madrid: Catedra.

Morín, E. (2008). La Cabeza Bien Puesta. Repensar La Reforma, Reformar El Pensamiento. Buenos Aires: Madrid: Catedra.

Morín, E. (2003). Los Siete Saberes Necesarios Para La Educación Del Futuro. Guayaquil: Santillana.

Morín, E. (2002). Sociología. Madrid: Tecnos.

Odom Y Káiser. (S.F De S.F De 1997). Conceptualización Del Desarrollo Y La Atención. Obtenido De Modelo Ecológico / Modelo Integral De Intervención: Http://Www.Sld.Cu/Galerias/Pdf/Sitios/Rehabilitacion_Temprana/Modelo_Ecologico_Y _Modelo_Integral_De_Intervencion.Pdf

Sander. (1990). Funcion De La Universidad.

Spaargaren,Mol Y Buttel . (S.F De S.F De 2000). El Fracaso De La Ecologización Del Mundo. Obtenido De Ética $\quad Y \quad$ Postecología: Https://Rua.Ua.Es/Dspace/Bitstream/10045/3094/1/Fracaso\%20ecologizacion\%20aledo. Pdf

Torre, D. L. (2013). La Universidad Que Necesitamos. Mexico: Juan Pablos Editor S.A. 